\title{
Can the Effectiveness of the Psychoanalytic Therapies Truly Be Proven?: The Effectiveness of the Psychoanalysis and Psychoanalytic Psychotherapy
}

\author{
Hye-Geum Kim, Eun-Jin Cheon, and Bon-Hoon Koo \\ Department of Psychiatry, College of Medicine, Yeungnam University, Daegu, Korea
}

\author{
정신분석적 정신치료의 효과는 검증될 수 있는가?: 정신분석 및 정신분석적 정신치료의 효과 \\ 김혜금 · 천은진 · 구본훈 \\ 영남대학교 의과대학 정신건강의학교실
}

The nature of psychoanalysis and long-term psychoanalytic psychotherapy makes it difficult to perform efficacy study including randomized controlled trial (RCT). Several RCTs are focused on the effectiveness of psychoanalytic psychotherapy; and more RCTs are conducted across multiple disorders for short-term psychoanalytic psychotherapy. As a result, there were more studies on psychoanalytic psychotherapy, as compared with that of cognitive behavioral therapy or pharmacotherapy. Moreover, psychoanalytic psychotherapy yielded significant benefits in terms of psychiatric symptoms but also the aspects of quality of life, adaptive capacities, and the reduction of maladaptive interpersonal behaviors. The psychotherapies of various types applying psychoanalytic theories were generally effective. Consequently, it can be inferred that long-term and intensive psychoanalysis is also effective.

Psychoanalysis 2016;27(3):81-91

KEY WORDS: Psychoanalysis · Psychoanalytic psychotherapy $\cdot$ Psychodynamic psychotherapy $\cdot$ Effectiveness.

\footnotetext{
Received: June 1, 2016 Revised: June 30, 2016 Accepted: July 1, 2016

Address for correspondence: Bon-Hoon Koo, MD, PhD

Department of Psychiatry, College of Medicine, Yeungnam University, 170 Hyeonchung-ro, Nam-gu, Daegu 42415, Korea

Tel: +82-53-620-3343, Fax: +82-53-629-0256, E-mail: vijnana@chol.com
}

\section{서 론}

최근의 임상 정신의학에서 치료는 대부분 근거 중심 의학 (evidence-based medicine)에 기반을 두고 있다. 이에 따라 대부분의 정신의학 교과서에서 각 질환의 치료 방침은 치료 효과에 대한 연구 결과들의 타당성을 바탕으로 이루어져 있 다. 현재 치료 효과를 판정할 때 가장 근거가 높은 연구는 무 작위 대조군 연구(randomized controlled trial, RCT)로 무 작위 할당이 연구의 내적 타당도(internal validity)에 필수적 으로 여겨지고 있다(Shadish 등 2002). 이에 다양한 RCT가 많이 나와 있는 약물치료는 정신의학에 있어 근거 중심 의 학의 최고 자리에 위치하고 있다. 또한 인지행동치료(cog-

This is an Open Access article distributed under the terms of the Creative Commons Attribution Non-Commercial License (http://creativecommons.org/licenses/by-nc/3.0) which permits unrestricted non-commercial use, distribution, and reproduction in any medium, provided the original work is properly cited. nitive-behavioral therapies, CBT)와 관련된 여러 치료의 연 구 결과가 꾸준히 발표되었으며, 전통적인 정신분석적 정신 치료에 비해 각 질환의 치료 우선 순위에 올라와 있다. 이에 반해 정신분석과 정신분석적 정신치료의 경우 무작위 할당 을 통한 RCT 연구에 여러 가지 제약을 가지고 있는 바, 결과 적으로 치료 우선 순위에서 밀려나고 있다. 미국 심리 협회 (American Psychological Association)에 의해 시행된 Task Force on Promotion and Dissemination of Psychological Procedures에 의하면 인지치료, 행동치료의 다양한 형태는 '확립 적 치료들(well-established treatments)'에 속하였으나, 단기 간 정신역동적 정신치료는 '잠정적 치료들(probably efficacious treatments)'로 보고하였다(Chambless 등 1998). 이렇게 과학 기술이 발달하고 이에 따라 사회적, 문화적 배경이 변 화함에 따라, 정신분석과 정신분석적 정신치료의 좀 더 과학 적인 검증이 요구되고 있다(Gunderson과 Gabbard 1999). 따라서, 본 연구에서는 먼저 정신분석과 정신분석적 정신치 
료가 현대 근거 중심 의학의 주류에서 검증을 받지 못하는 이유에 대해 살펴 보고, 정신분석과 정신분석적 정신치료의 효과를 검증한 연구들을 체계적으로 검토해 보고자 한다.

\section{본 론}

\section{정신분석 및 정신분석적 정신치료에서 무작위 대조군 연구가 부족한 이유}

현재 RCT는 치료 방법을 평가할 때 가장 높은 근거를 보 여 주는 'gold standard' 방법이다. RCT는 대상군을 각기 다 른 치료군으로 무작위 할당하는 방법인데, 이는 이미 전에 존재하는 대상자 간의 차이가 동등하게 배분되도록 하여 적 용된 각 치료의 효과만을 비교하는 것을 목적으로 한다(Shadish 등 2002). 이에 근거하여 정신치료적 방법들의 실험적 연구 또한 오직 RCT로 이루어져야 한다면, 많은 방법적 제 한점이 발생한다. 첫째, 기존 RCT에서는 무작위 할당된 치 료군과 대조군을 비교하는데, 이 대조군은 흔히 치료 대기 군, 위약군, 또는 이미 효과가 잘 검증된 다른 치료군이다(Chambless와 Hollon 1998; Chambless와 Ollendick 2001). 그런 데 보통 수년 이상 걸리는 정신분석 및 정신분석적 정신치료 에서는 그렇게 긴 기간 동안 치료 대기 상태 또는 위약군으 로 있을 수 있는 대조군은 존재하기 힘들며, 충분히 신뢰 가 능한 동일한 대조군을 유지하기는 힘들다(Seligman 1995; Leichsenring 2005). 둘째, 임상 현장에서 정신분석을 받기 를 원하는 환자군과 좀 더 단기간의 치료를 받기를 원하는 환자군은 특정한 성격적 특징들로 차이를 보인다(Rudolf 등 1994). 그렇기 때문에 환자를 정신분석적 치료군과 대조군 으로 무작위로 할당하는 것은 환자와 적합한 치료의 연결을 방해하며, 결과적으로 연구의 결과로 표현되는 치료의 효과 는 잘못될 가능성이 많다. 이에 Leichsenring(2005)은 “정신 분석적 치료의 무작위 비교 연구의 결과는 통상적으로 정신 분석적 치료를 받는 환자들에게는 유효하지 않다."고 주장 하였다. 셋째로, RCT는 모든 치료군에게 동등하게 적용될 수 있는 치료 매뉴얼과 각 질환에 대한 특정 치료 방법이 요 구된다(Beutler 1998; Leichsenring 2004). 하지만 정신분석 및 정신분석적 정신치료는 각 환자에 맞추어 고도로 개별화 된 치료이며, 수년이라는 장기간 동안 진행되기 때문에 치료 매뉴얼이 존재하기 어렵다. 이것은 정신분석적 이론과 인간 의 마음의 특성에서 기인한 것으로 특히 정신분석의 가장 중요한 도구인 자유연상은 각 개인의 마음에 떠오르는 생각 과 감정으로서 매뉴얼화가 될 수 없다. 마찬가지로, 환자가 자신의 내면을 보기 어려워해서 발생하는 저항과 치료자에 대한 전이, 그리고 치료자의 역전이 등도 개별적인 환자와
분석가마다 모두 다르고, 또한 환자와 치료자의 관계 및 상 호작용도 각 세션마다 다르기 때문에 이를 객관적으로 측정 하는 것은 거의 불가능하다. 또 정신치료는 내과 질환에서 주 가 되는 약물치료와 달라서 각기 다른 환경에서 비슷한 효과 를 내기 어렵다. 그렇기 때문에 내과 질환에 최적화되어 있는 실험적 연구들은 복잡한 정신 질환 상태에서는 적합하지 못 하다(Leichsenring 2005). 넷째, 타당성이 입증된 치료들 (empirically validated treatments)은 각 질병과 증상에 초점 을 맞추었으며, 대개 약물치료나 $\mathrm{CBT}$ 의 경우 증상의 호전 이 목적이지만 정신분석의 경우 치료의 목적은 개개인에 따 라 다를 수 있고, 심지어 정신분석의 경우, 분석의 끝은 어떻 게 될지 알 수 없다고도 하였다(Lipton 1961). 이러한 이유 로 지금까지는 정신분석 및 정신분석적 치료에 대한 RCT가 부족하였으며, 일각에서는 RCT가 정신치료의 효과를 보여 주기에 적합한 연구 방법이 아니라는 주장도 있다(Leichsenring 2005). 여러 연구자들은 장기적인 정신역동적 치료에 는 자연관찰 연구(naturalistic studies)를 이용하여 치료의 효 과(effectiveness)를 살펴보는 것이 RCT를 이용해서 효능(efficacy)을 검증하는 것보다 적절하다고 하였다(Seligman 1995; Leichsenring 2004; Westen 등 2004). 즉, 효능(efficacy)은 이 상적인 실험적 상황에서 특정한 중재의 이로운 정도를 말하 며, 효과(effectiveness)는 일상적인 실제 임상 상황에서 특 정 인구에게 어떤 중재가 무엇을 해 줄 수 있는지를 말해 준 다. 이러한 맥락에서 정신치료의 효과를 밝히는 연구에 있어 서 효과 연구(effectiveness studies)가 결코 치료 효과를 과대 평가하지는 않는다는 결과도 있었다(Benson과 Hartz 2000; Concato 등 2000; Leichsenring 2005).

\section{정신분석 및 정신분석적 치료의 효과 검증 연구}

위에서 살펴본 정신분석 및 정신분석적 치료의 특성들로 인하여 효과 검증 연구에 어려움이 있으나 그 효과를 과학적 으로 검증하고자 하는 시도가 있었다. 정신분석 및 장기 역동 정신치료의 정의는 Gunderson과 Gabbard(1999)에 의하면 "치료자와 환자의 상호작용에 초점을 두고, 이에 대한 치료 자의 정교한 이해를 바탕으로 하는 적절한 시기의 전이와 저 항의 해석이 이루어지는 치료”라고 하였다. 그리고 장기 역 동 정신치료에 정해진 치료 기간은 없는 편이며, Lamb(2004) 에 의하면 20개 이상의 다른 정의가 있었다고 보고하였는 데, 짧게는 3 개월에서부터 최대 20년의 넓은 범위를 가지고 있었다. 이 논문에서는 통상적으로 많은 연구에서 정의한 것 을 적용하여 6개월 이상 또는 24세션 이상의 역동적 정신치 료를 장기 역동 정신치료로 간주하였다(Gabbard 2010). 그 리고 그 이하의 기간 및 세션 동안 시행된 정신역동적 치료 
는 단기 역동 정신치료로 간주하였다.

\section{정신분석의 효과 검증 연구}

정신분석의 효과를 검증하기 위해 RCT를 대신할 수 있는 다른 방법으로 내적 타당도를 저해할 수 있는 요인들을 최 대한 교정하려는 노력이 동반된 전향적 준실험설계 연구(quasi-experimental studies)가 있다. Nathan과 Gorman(2015) 에 의해 제안된 RCT에서의 효능의 근거 level I에 상응하는 근거를 가진다고 주장되기도 하였으며(Leichsenring 2005), 정신분석 효과 검증에 자주 이용되었다. Duihrssen과 Jorswieck(1965)에 의한 연구에서는 5년 동안 대형 건강 보험 회사의 자료를 토대로 정신분석의 전후를 비교하였는데, 정 신분석을 받은 125 명의 치료군과 정신분석을 받지 않은 환 자들 중 무작위로 선정된 100명의 대조군을 비교하였다. 연 구 결과 정신분석군에서 병원 내원 일수가 적었고 건강 서비 스 비용 지출이 적었다. Rudolf 등(1994)은 정신분석, 평균 60세션의 중등도 길이의 정신역동적 정신치료, 그리고 정신 역동적 입원 환자의 치료를 비교하였다. 이 세 그룹은 진단 과 질환의 심각도가 비슷하였다. 그 결과 정신분석의 $76 \%$ 에 서 임상적으로 유의한 호전을 보였으며, 이는 정신역동적 정 신치료의 $55 \%$, 정신역동적 입원 환자의 치료 $50 \%$ 의 호전 수준에 비해 우월하였다. Sandell 등(2002)은 정신분석과 장 기 정신역동적 정신치료의 효과를 밝히기 위해 치료 이전의 군 간 차이는 통계적으로 보정한 후 연구를 진행하였다. 그 결과 정신분석은 1.55 의 큰 효과 크기(effect size)를 보여 주었 으며, 이와 상응하는 평균 233세션을 진행한 장기 정신역동적 정신치료의 효과 크기는 0.60이었다(Blomberg 등 2001).

Bachrach 등(1991)의 보고에 따르면 정신분석의 효능(efficacy)은 60 91\%의 범위에 이른다고 하였는데, 이는 치료를 유지하는 군에 한해서였고, 일각에서는 선택 오류(selection bias)가 있지 않은지 의문을 제기하기도 하였다. 하지만 연 구자는 선택 오류는 임상 현장에서 자연적으로 일어나는 현 상이며, 정신분석은 장기적으로 치료에서 치료적 이득을 얻 을 수 있는 환자군을 위한 치료라고 주장했다(Doidge 1997). 정신분석에 대한 첫 번째 전향적 연구로 알려진 Menninger Foundation Psychotherapy Research Project는 1954년 시 작되어 30년간 진행되었으며, 주로 정신분석의 치료에 영향 을 주는 인자와 정신분석으로 가장 큰 이득을 보이는 환자 군을 밝히는 것이 목적이었다. 전반적으로 $60 \%$ 의 호전을 보 였는데, 대인 관계와 관련된 문제가 주된 환자군과 일차적 회피성 방어(primarily avoidant defenses)를 사용하는 환자 군은 정신분석보다는 정신치료가 나았고, 반대로 자기 인식 (self-definition), 자율성(autonomy), 그리고 자기 가치(self- worth)와 관련된 문제가 주된 환자군, 그리고 일차적 내사적 방어(primarily introjective defenses)를 사용하는 환자군에 서는 정신분석이 나았다(Kernberg 등 1972; Wallerstein 2000). 이 외에도 몇 개의 연구 프로젝트들이 있었다. 1959년 시작된 The Columbia University Research Project는 정신 분석가 과정에 있는 수련생(candidate)에 의한 700명의 정신 분석, 그리고 885 명의 정신치료 사례가 포함되었다. 최종 분 석은 295사례였으며, 그 결과 $91 \%$ 에서 호전을 보였으며, 분 석적 과정(analytic process)이 나타난 군은 $43 \%$ 에 불과하였 지만, 분석적 과정이 없어도 치료적 이득은 있었다(Weber 등 1985c; Weber 등 1985a). 이후 이어진 The Second Columbia University Study에서는 125 명의 정신분석적 정신치료 환자 군과 35 명의 정신분석 환자군을 대상으로 분석하였을 때, 정 신분석적 정신치료군의 전반적 호전율은 $79 \%$ 였으며, 3 명을 제외하고는 만족 또는 매우 만족하였다. 치료 종결까지 유지한 군에서는 $96 \%$ 의 호전을 보였으며, 52\%에서 많은 호전(much improved), $44 \%$ 에서는 호전(improved)을 보이며, $1 / 3$ 에서 는 최대한의 이득(maximum benefit)을 보였다. 35 명의 정 신분석군에서는 치료 기간의 길이는 치료적 이득뿐 아니라 분석적 과정(analytic process)의 발달과도 연관되었다(Weber 등 1985b). 1959년부터 1966년까지 시행된 또 다른 연구 로 The Boston Psychoanalytic Institute Prediction Studies 가 있는데, 정신분석가 과정에 있는 수련생(candidate)에 의 해 치료된 130명의 환자를 대상으로 하였다. $75 \%$ 의 환자군 에서 적어도 중등도 이상의 호전을 보였으며, 치료 기간의 길 이는 좀 더 나은 치료 결과와 관련이 되었으며, 가장 높은 호 전도는 상호 간의 합의를 통해 종결한 군에서 보였다(Erle 1979; Erle와 Goldberg 1984).

정신분석의 효과 크기(effect size)에 대한 연구들은 다음 과 같다. 효과 크기를 해석할 때 큰 효과라고 간주되는 0.80 이 상의 효과(Cohen 1988)를 보인다고 보고한 연구에는 Duihrssen과 Jorswieck(1965), Rudolf 등(1994), Luborsky 등(1999), Sandell(1999), Sandell 등(2000), Brockmann 등(2003), Rudolf 등(2004), 그리고 Leichsenring 등(2005)에 의한 연구들이 있 다. 또한 정신분석의 효과는 증상의 경감뿐 아니라 자존감이 나 대인 관계와 같은 삶의 질 측면 및 전반적인 안녕 상태 (well-being)의 개선에도 의미가 있었다(Leuzinger-Bohleber 등 2003). 뿐만 아니라 병가(sick leave) 기간의 감소 및 내과 적 협진 횟수도 유의한 감소가 있었다고 보고된 연구도 있었 다(Beutel 등 2004). 상기 소개된 Menninger Psychotherapy Research Project의 재평가로서, Blatt와 Shahar(2004)는 정 신분석이 적응적인 대인 관계 능력을 개발하고 비적응적인 대인 관계 행동을 줄이는 데 유의한 기여를 한다고 보고하였 
으며, 이는 특히 보다 자기 투영적인(self-reflective) 환자들 에서 두드러졌다고 하였다.

\section{단기 역동 정신치료의 효과 검증 연구}

단기 역동 정신치료는 특성상 단기에 치료가 끝나기 때문 에 RCT 연구가 보다 가능할 수 있다. 따라서 정신분석이나 장 기 역동 정신치료에 비해 여러 가지 질환들에 RCT가 많이 진행된 편이었다. 우선 우울증에 대한 RCT들로는, Thompson 등(1987)에 의한 연구에서 24명의 단기 역동 정신치료군 과 행동치료군 25명, CBT군 27명, 치료 대기군 19명을 비교 하였고, Shapiro 등(1994)에 의한 연구에서 단기 역동 정신 치료군 58명과 $\mathrm{CBT}$ 치료군 59 명을 비교하였으며, 같은 해 Gallagher-Thompson과 Steffen(1994), Barkham 등(1996) 도 단기 역동 정신치료군과 $\mathrm{CBT}$ 치료군을 비교하였다. 이 연구들에 대한 메타 분석 결과, 역동 정신치료는 $\mathrm{CBT}$ 와 동 등한 효과를 보여 주었으며, 이는 우울 증상뿐 아니라 일반 정신과적 증상과 사회적 기능에도 적용되었다(Leichsenring 등 2014). 최근 연구로 Maina 등(2005)은 단기 역동 정신치료 군 10 명과 지지적 치료군 10 명 및 치료 대기군 10 명을 비교 하였고, 결과 두 치료군이 치료 대기군보다 효과적이었으며, 6개월 경과 관찰 이후 역동 정신치료군이 지지적 치료군보 다 우수하였다. Salminen 등(2008)은 26명의 단기 역동 정신 치료군과 25명의 fluoxetine 복용군을 비교하였는데, 두 군 모두 우울 증상과 기능적 측면을 호전시키는 데 동등하게 효과적이었다. 최근 메타 분석(Driessen 등 2010)에서는 역 동 정신치료군이 대조군에 비해 우월하였다고 보고하였고, 집단 치료를 제외한 분석 시 어떤 하나의 치료가 우월하지 는 않았다고 보고하였다(Abbass와 Driessen 2010). 한편, 효 과가 없다고 보고한 논문도 있다. 최근 RCT에서는 단기 역 동치료군과 약물치료군 및 위약군을 비교하였는데, 두 치료 군은 동등한 효과를 보였지만, 위약군보다 유의한 우월성을 보여 주진 못하였다고 보고하였다(Barber 등 2012). 같은 해 Johansson 등(2012)은 역동 정신치료에 기반을 둔 인터넷 기반의 자조 치료를 구조화된 지지 치료군과 비교하였다. 그 결과 역동 정신치료에 기반을 둔 인터넷 기반의 자조 치료 가 더 우수한 효과를 보여 주었고, 이 효과는 10 개월 이상 유 지되었다.

그 다음으로 불안 장애들에 대한 단기 역동 정신치료의 $\mathrm{RCT}$ 로는, Knijnik 등(2004)의 사회 공포증 환자를 대상으로 대조군과 비교 시 단기 역동 정신치료가 우월하다고 보고하 였고, Crits-Christoph 등(2005)은 범불안 장애 환자를 대상 으로 지지 치료와 비교한 결과 증상의 관해율에서 단기 역 동 정신치료가 지지 치료보다 우월하였다. Milrod 등(2007)
은 공황장애 환자군을 $\mathrm{CBT}$ 군과 비교 시, 단기 역동 정신치 료가 우수하였다고 보고하였다. Brom 등(1989)은 외상 후 스 트레스 장애(post-traumatic stress disorder, PTSD)를 대상 으로 29명의 단기 역동 정신치료군과 탈감작 치료군 31명 및 최면 치료군 29 명을 비교한 결과 모든 치료가 동등하게 효 과적이라고 보고하였다. 하지만 이후 이 연구에 대한 비판이 있어 왔는데, Ehlers 등(2010)은 어느 치료도 치료 대기군보 다 우월하지 않다고 주장하며, 결과를 판정하는 척도에 따라 서 결과가 달라진다는 점 등을 내세웠다. 지금까지는 PTSD 에 대한 RCT가 부족한 실정으로, $\mathrm{PTSD}$ 의 역동적 정신치료 에 대한 효과를 결론짓기에는 이른 것으로 판단된다(Leichsenring 등 2014).

신체화 장애를 대상으로 한 RCT는 Guthrie 등(1991), Hamilton 등(2000), Creed 등(2003), Sattel 등(2012)에 의한 연 구들이 있으며, 각 내과적인 일상적인 치료 및 지지적 치료와 단기 역동 정신치료를 비교하였다. 그 결과 모두 단기 역동 정신치료군에서 더 우수한 효과를 보여 주었으며, Abbass 등 (2009)에 의한 종설에서 신체화 장애 환자들을 대상으로 한 단기 역동 정신치료는 신체적 증상, 정신과적 증상과 사회적 적응에도 유의한 효과가 있다고 보고하였으며, 이 효과는 장 기적으로 유지된다고도 하였다.

식이장애에서 단기 역동 정신치료의 효과를 살펴본 RCT 로는, 우선 Fairburn 등(1986), Garner 등(1993)이 신경성 대 식증(bulimia nervosa, $\mathrm{BN}$ ) 환자를 대상으로 $\mathrm{CBT}$ 치료군과 비교한 연구가 있다. 이 연구들의 결과상 단기 역동 정신치료 는 유의하고 안정적인 호전을 보여 주었는데, 결과 척도에 따 라서 $\mathrm{CBT}$ 가 우월한 듯 보이는 부분도 있으나 이후 경과 관 찰한 연구(Fairburn 등 1995)에서는 단기 역동 정신치료가 우 수하였다고 보고하였다. 한편, 신경성 거식증(anorexia nervosa, AN) 환자에 대한 근거 기반의 치료는 현재 많이 부족 한 실정이다(Fairburn 2005). 하나의 단기 역동적 정신치료 에 대한 하나의 RCT가 있는데, Gowers 등(1994)이 신경성 거식증 환자를 대상으로 일상적인 치료와 비교한 연구에서, 체중과 체질량지수가 대조군에 비해 유의한 호전이 있었다 고 보고하였다. 하지만 아직 $\mathrm{AN}$ 에 대한 연구는 부족한 편으 로 좀 더 많은 연구가 필요한 것으로 판단된다. 그리고 Tasca 등(2006)이 폭식 장애(binge eating disorder) 환자를 대상으 로 집단 $\mathrm{CBT}$ 및 치료 대기군과 비교한 연구를 보고하였으 며, 단기 역동 치료군이 $\mathrm{CBT}$ 군과 동등하게 치료 대기군보 다 효과가 있었다고 보고하였다.

물질과 관련된 장애들(substance-related disorders)에 대 한 단기 역동 정신치료의 RCT는 Woody 등 $(1983 ; 1990)$ 에 의한 opiate 의존 환자들을 대상으로 하여 약물 상담과 단기 
역동 정신치료를 병합한 군을 약물 상담 단독군 및 약물 상 담과 CBT의 병합군과 비교한 연구, Sandahl 등(1998)에 의 한 알코올 의존 환자들을 대상으로 CBT군과 비교한 연구가 있다. 두 연구 모두에서 단기 역동적 정신치료는 $\mathrm{CBT}$ 와 동 등하거나 우월한 치료 효과를 보여 주었다.

경계선 인격장애(borderline personality disorder, BPD)에 서 역동 정신치료의 효과에 대한 RCT는 보다 장기적인 연구 들도 많이 있다. BPD에 대한 단기 역동 정신치료의 RCT는 Munroe-Blum과 Marziali(1995)에 의해 발표된 연구로, 대 인 관계 집단 치료군과 비교한 17 세션 동안 진행된 연구가 있 었다. 연구 결과 단기 역동 정신치료가 경계선과 관련된 증 상들(borderline-related symptoms), 일반적인 정신과적 증 상들, 그리고 우울 증상에서 유의한 호전을 보여 주었으며, 대인 관계 집단 치료군과 비교 시에는 유의한 차이를 발견하 지는 못했다. C군 인격장애에 대한 RCT들도 보다 장기적인 역동 정신치료 연구들이 많으며, 단기 역동 정신치료 연구 중에는 Emmelkamp 등(2006)에 의한 회피성 인격장애 환자 23명을 대상으로 CBT군 21명 및 치료 대기군 18명과 비교 한 연구가 있다. 이 연구에서는 단기 역동 정신치료가 $\mathrm{CBT}$ 만 큼이나 치료 대기군보다 효과적이라고 보고하였는데, 경과 관찰 이후에는 치료 대기군보다 우수한 결과를 발견할 수 없었는데 적은 수의 대상자군이 영향이 있었을 것으로 평가 되었다(Leichsenring과 Leibing 2007). 최근 발표된 메타 분 석(Town 등 2011)에서는 인격장애 환자들을 대상으로 한 단기 역동 정신치료의 효과를 보고하였는데, 저자들은 역동 정신치료가 효과적인 경험적으로 지지된 치료(empirically supported treatment)가 될 수 있음을 주장하였다.

\section{장기적인 역동 정신치료 효과 검증 연구}

위에서 정의하였듯, 6 개월 이상 또는 24 세션 이상의 역동 적 정신치료를 장기 역동 정신치료로 간주할 때 장기 역동 정신치료에 대한 연구들도 있다. Luborsky 등(1988)에 의해 시행된 The Penn Psychotherapy Research Projects는 1968 년 시작하여 5년간 진행되었다. 이 연구에서는 매뉴얼에 따 른 정신분석적 정신치료를 평균 32세션 진행하였으며, 총 73 명이 포함되었다. 연구 결과 전반적인 호전율은 $92 \%$ 로, 중등도의 호전이 $43 \%$, 일부 호전이 $27 \%$, 큰 호전이 $22 \%$ 에서 나타났다. 그 외에 장기 역동 정신치료에 대한 RCT는 다음 과 같다.

우울 및 불안 장애 환자들을 대상으로 하여, Knekt 등(2008) 은 단기 역동 정신치료 및 장기 역동 정신치료, 그리고 해결중심 치료(solution-focused therapy)군을 비교하였다. 그 결과 단기 역동 정신치료가 장기 역동 정신치료보다 첫 1년
동안은 보다 효과적이었지만, 2 년 이후 경과 관찰을 하였을 시 두 군의 효과 차이가 사라졌으며, 3 년 후에는 장기 역동 정신치료군이 보다 효과적이었다고 보고하였다. 이후 Bressi 등(2010)에 의한 연구에서는 일상적인 치료군을 대조군으로 하여 진행된 연구로 장기 역동 정신치료군에서 보다 나은 치료 결과를 보고하였다.

불안 장애 중 사회 공포증 환자들을 대상으로 한 장기 역동 정신치료의 RCT는 Bögels 등(2014)에 의한 연구로 CBT군 과 비교한 결과, $\mathrm{CBT}$ 만큼이나 효과적임을 밝혔다.

2013년 발표된 대규모의 다기관 연구(Leichsenring 등 2013) 에서는 총 495명의 사회공포증 환자들을 대상으로 CBT군, 장기 역동 정신치료군, 그리고 치료 대기군으로 무작위 배정 하였다. 그 결과 두 치료군은 모두 효과적이었는데, 치료 반 응률에서는 차이가 없었고, 관해율에서는 $\mathrm{CBT}$ 에서 조금 더 유리했으나 그 차이는 미미했다. 범불안 장애 환자군을 대상 으로 한 장기 역동 정신치료의 RCT는 Leichsenring 등(2009) 의 연구에서 $\mathrm{CBT}$ 군과 비교하였고, 그 결과 일차적 결과 지 표에서는 두 군이 동등하게 효과가 있었는데, 이차적 결과 지 표에서는 몇 개의 항목에서는 CBT가 우월하였다고 보고하 였으나 대상자 수가 적었기에 해석에 제한이 있었다. 신체형 통증 장애 환자군을 대상으로 한 Monsen과 Monsen(2000) 의 연구에서는 장기 역동 정신치료와 일상적 치료군 및 치료 를 하지 않는 군과 비교하였을 때, 역동 정신치료군이 대조 군들에 비해 효과가 우수하였고, 특히 통증, 정신과적 증상, 대인 관계 문제, 그리고 정동 자각(affect consciousness)에서 효과적이었다고 보고하였다.

$\mathrm{AN}$ 과 $\mathrm{BN}$ 을 포함한 식이장애에 대한 장기 역동 정신치료 의 RCT는 Bachar 등(1999)에 의한 연구가 있는데, 인지치료 및 영양 상담을 시행한 군에 비해서는 우수한 효과를 보이는 결과를 보여줬다. AN 환자군을 대상으로 한 연구에는 Dare 등(2001)의 연구가 있는데, 인지치료군, 가족치료군, 그리고 일상적인 치료군과 비교하였을 때, 장기 역동 정신치료와 가 족치료가 다른 치료들에 비해 효과적이었으나, 호전 정도가 미약하였고 경과 관찰 중에 다시 악화된 군도 있었다. 아직, $\mathrm{AN}$ 에 대한 역동 정신치료에 대한 효과에 대한 연구는 더 필 요해 보인다. 그 외 물질과 관련된 장애로 cocaine 의존에 대 한 장기 역동 정신치료에 대한 RCT는 Crits-Christoph 등 (2001)에 의한 연구, opiate 의존에 대해서는 Woody 등(1995)의 연구가 있다. 두 연구 모두에서 장기 역동 정신치료에서 CBT 와 동등한 정도의 효과가 있었으며, 그 효과가 장기적으로 유 지되었다.

경계선 인격장애(BPD)를 포함한 인격장애에 대한 장기 역동 정신치료의 RCT 연구 결과는 상대적으로 많은 편이다. 
BPD에 대한 연구는 Bateman과 Fonagy(1999; 2001), Clarkin 등(2007), Giesen-Bloo 등(2006), Gregory 등(2008)에 의한 연구들이 있다. 모든 결과에서 장기 역동 정신치료가 일반적인 정신과적 치료에 비해 효과적이었으며, 치료 직후 뿐 아니라 1년 이상 경과 관찰을 하고 난 뒤에도 효과가 유지 되었다(Leichsenring 등 2014). C형 인격장애에 대한 RCT는 Svartberg 등(2004)에 의한 연구에서는 CBT와 비교 시 동등 하게 효과적이었으며, 증상뿐 아니라 대인 관계 문제들, 그 리고 핵심 인격 병리(core personality pathology)에 효과적이 었고, 그 효과는 24개월 동안 안정적으로 유지되었다. 이후 Muran 등(2005)에 의한 연구에서는 장기 역동 정신치료를 단기 관계 치료(brief relational therapy), 그리고 CBT와 비교 하였다. 결과 각 치료군 간의 유의한 차이는 없었으나, 치료 종결 시점에서 장기 역동 정신치료보다 다른 두 치료에서 우 월한 하나의 결과 항목(환자 보고식 불평들)이 있었지만 경 과 관찰 이후에는 사라졌다. 그 외의 복합적인 인격장애군에 대한 연구들로 Hellerstein 등(1998), Abbass 등(2008), Winston 등(1994), Vinnars 등(2005)의 연구가 있다. Høglend 등 (2006)은 장기적 역동 정신치료를 세분하여 전이(transference) 해석을 하는 군과 하지 않는 군으로 나누어 무작위 배 정한 뒤 결과를 확인하였더니, 성숙한 대상 관계를 가지지 못한 환자군에서는 전이 해석이 도움이 되는 양상을 보였다. 인격장애에 대한 역동적 정신치료의 효과에 대한 메타 분석 (Leichsenring과 Leibing 2003) 결과도 있었다. 그 결과에서 는 인격장애에서 역동적 정신치료와 $\mathrm{CBT}$ 의 효과를 살펴보 면, 역동 정신치료에서 큰 효과 크기(effect size)를 보여 주었 는데, 동반된 증상뿐만 아니라 핵심적 인격 병리에도 효과적 이었다고 보고하였다. 그리고 이는 특히 BPD 환자군에서 두 드러진 효과를 보여 주었다고도 하였다.

\section{기타 최근의 다양한 정신치료 효과 연구}

최근에는 정신분석 이론 및 방법을 적용한 좀 더 다양한 형태의 정신치료들이 시행되고 있으며, 이러한 정신치료들에 대한 효과를 검증하고자 하는 연구들도 발표되고 있다. 이러 한 정신치료들에는 대인 관계 정신치료(interpersonal psychotherapy, IPT), 전이 중심 정신치료(transference focused psychotherapy, TFP), 정신화 중심 치료(mentalization based treatment, MBT), 공황 중심 정신역동 정신치료(panic-focused psychodynamic psychotherapy, PFPP) 등이 있다. 우 선 IPT에 대한 연구들을 살펴보면, 최근 한 메타 분석(Cuijpers 등 2008)에서 성인 우울증에서 CBT와 역동 정신치료, $\mathrm{IPT}$, 그리고 다른 형태의 정신치료의 효과를 검토하였으며, 그 결과 IPT가 조금 더 효과적이었고 그 외 어느 치료가 더
우월하지 않았다. 한 RCT(Zobel 등 2011)에서는 약물치료 중인 우울증 환자들을 대상으로 IPT를 병합하는 군 또는 일 반적 임상 관리군(clinical management)으로 무작위 배정하 였고, 5 주간 진행 이후 평가해 보았을 때, IPT 병합군이 좀 더 빠른 증상의 호전을 보였으며 유의하게 높은 지속적인 관해 율을 보였다. 가장 최근에 보고된 RCT(Ekeblad 등 2016)에 서는 지역사회 기반의 정신건강의학과 외래에서 주요 우울 장애 환자들을 대상으로 $\mathrm{CBT}$ 군과 IPT군으로 무작위 배정 하여 14 세션을 진행하였다. 그 결과 IPT 환자군의 $53.5 \%$, CBT 환자군의 $51.0 \%$ 에서 유의한 호전이 있었고, IPT 환자 군의 $20.9 \%$, CBT군의 $19.1 \%$ 에서 우울증이 회복되었다고(recovered) 보고하였다. 이 결과는 항우울제의 복용에 대한 변 수를 보정하고도 변함없었다고 하며, 저자들은 IPT가 CBT 보다 결코 열등하지 않다고 주장하였다.

다음으로 TFP에 대한 최근 RCT(Doering 등 2010)에서는 Kernberg의 모델(Kernberg 등 2008)을 바탕으로 BPD 여자 환자 104명을 대상으로 $\mathrm{TFP}$ 군 또는 경험 있는 지역사회 정 신치료가(experienced community psychotherapist)에 무작 위 배정하여 1 년간 치료하였다. 그 결과 $\mathrm{TFP}$ 군에서 경계선 정신병리, 정신사회적 기능, 인격 조직화, 정신과적 입원, 그 리고 탈락률(drop out)에서 더 우월하였다고 보고하였다. 또 다른 RCT(Clarkin 등 2007)는 TFP와 변증법적 행동 치료 (dialectical behavior therapy, DBT), 그리고 정신역동적 지 지적 정신치료를 비교하였다. 그 결과 모든 치료군에서 전반 적인 호전을 보였으나, TFP가 다른 군에서 보이지 않은 호 전을 보여 준 부분들이 있었는데, TFP군이 불안정한 애착군 에서 안정적 애착군으로 이행이 유의하게 많았고, 정신화 능 력(mentalization capacity)과 서술적 긴밀성(narrative coherence)에서 유의한 변화를 보였다. 또한 다른 군과 달리 $\mathrm{TFP}$ 군만이 충동성, 예민성, 언어적 폭력성, 그리고 직접적 폭행(assault)에서 유의한 호전이 있었고, TFP군과 DBT군, 두 군만이 자살위험성이 감소하였다고 보고하였다.

$\mathrm{MBT}$ 에 대한 효과 검증 연구도 현재 다수 진행되고 있으 며, 대표적으로 Bateman과 Fonagy(2009)에 의한 BPD를 대 상으로 한 RCT 결과가 있다. 매뉴얼에 따른 구조화된 임상 적 관리(manual-driven structured clinical management)와 비교 시 $\mathrm{MBT}$ 군은 자살, 자해 행동, 그리고 정신과적 입원뿐 아니라 우울, 전반적인 불편감, 대인 관계적 기능에서도 유 의한 호전을 보였다고 보고하였다. 또 다른 RCT(Rossouw 와 Fonagy 2012)로는 자해를 하는 청소년들을 대상으로 일 상적인 치료군과 MBT군을 비교했을 때, MBT군이 자해 행 동 및 우울증에서 좀 더 효과적이었다고 보고하였으며, 또 다른 RCT(Jørgensen 등 2013)에서는 BPD를 대상으로 MBT 
와 지지적 정신치료를 비교했을 때, 두 군 모두 전반적인 호 전을 보여 주었으며 전반적 기능 척도(global assessment of functioning)에서는 MBT군만이 호전을 보여 주었고 전반적 으로 MBT군에서 회복이 더 많은 경향으로 보고하였다.

마지막으로 PFPP에 대한 연구는 개방형 임상 연구 및 RCT가 1997년부터 2005년까지 Weill Cornell Medical College에서 진행이 되었다. 개방형 연구 결과 PFPP를 끝낸 17 명 중 16 명이 치료에 반응을 보였으며, 임상적 호전은 6개월 경과 관찰 중에도 계속 유지되었다. 이후 진행된 RCT에서 는 응용 이완 훈련(applied relaxation training, ART)을 대조 군으로 잡아 시행하였는데, ART군에 비해 PFPP군이 보다 효과적이었고, 증상뿐 아니라 정신사회적 기능의 호전도 보 였다. 현재는 CBT, PFPP, 그리고 ART에 대한 대규모의 RCT 가 진행 중에 있다(Milrod 등 2007; Busch 등 2009).

\section{정신치료 효과 연구에 대한 기타 연구 이슈들}

정신치료에 대해 위에서 주로 살펴본 RCT를 비롯한 효과 검증 연구들은 치료의 결과에 초점이 맞추어져 있으며, 역동 정신치료의 과정과 관련된 변수들은 살펴볼 수 없다. 하지 만, 정신치료적 과정(psychotherapeutic processes)에 대한 연구는 정신역동적 치료에서 변화의 기전을 고려하는 데 중요 한 자료를 제공한다(Leichsenring 2005). Ablon과 Jones(2005) 는 분석적 과정(analytic process)은 조작 가능하고(operationalized) 정량적으로 평가(quantitatively assessed)될 수 있 다고 주장하였다. 이들은 Psychotherapy Process Q-set(PQS) (Jones 2000)라는 평가 도구를 이용하여 정신치료 과정의 정 량적 분석을 시도하였다. 실제로 무작위로 선정된 환자의 치 료 비디오 테이프나 치료 기록(transcripts)을 보면서 $\mathrm{PQS}$ 를 이용하여 정신치료 과정을 평가하면서 치료적 작용(therapeutic action)이 어떻게 이루어지는지를 확인하였다. 그 결 과 분석적 과정은 일률적인 과정이 아니고, 각 환자와 분석 가의 조합은 각 치료마다 독특하고 뚜렷이 구별이 가능한 패턴의 상호작용을 가지며, 이러한 패턴이 치료 결과와 연관 된다고 하였다. 또한 치료의 영향은 치료자에서 환자로 향하 는 한 방향(one direction)이 아니고 두 사람의 상호작용(dyadic)으로 나타나며, 경험(the experience), 인식(recognition), 그리고 이해(comprehension) 등 치료적 작용의 기초적인 구 성요소로 생각되는 모든 것들이 반복된 상호 작용의 패턴을 보인다고 하였다. Blatt와 Auerbach(2003) 또한 정신치료적 변화에 대한 정신역동적 평가를 시도하였다. 이들은 기존의 평가 척도들을 이용하였는데, 여기에는 자기와 의미 있는 대 상들의 구조와 표현 요소(content of descriptions)들을 평가 하는 방법으로 Differentiation-Relatedness Scale, Conceptual
Level Scale, Qualitative-Thematic Scales를 포함하는 Object Relations Inventory, 자신과 상대방의 마음의 상태를 파악하는 마음 이론(theory of mind) 및 반사 기능(reflective functioning, RF)의 수준을 평가하는 RF 등이 포함된다. 또 한 이들은 정신치료 연구는 각 환자들에 따라 개별화될 필 요가 있으며, 각 환자가 치료 과정에서 어떻게 기여하고 있 는지를 체계적으로 평가할 필요가 있다고 주장하였다. 즉 환 자의 특성과 치료 변수들의 상호 작용이 중요하며, 각기 다 른 종류의 치료는 각각 다른 환자들에게 좀 더 효과가 있거 나 없을 수 있는데, 치료적 과정은 동등하게 바람직한 결과 를 보이지만, 각기 다르게 표현될 수 있다고 하였다. 결과적 으로 이들은 의존적인(anaclitic) 환자들은 정신분석보다 정신 치료에서 유의한 긍정적 변화가 있었고, 내사적(introjective) 환자들은 반대로 정신치료보다는 정신분석이 나았다고 보 고하였다(Blatt 1992; Blatt와 Auerbach 2003).

이 외에 정신치료의 과정과 관련되어 연구 가능한 이슈들 에는 다음과 같은 것들이 있다. 첫째, 정신역동적 정신치료 의 결과는 정신치료적 기술과 치료자의 능숙함(psychotherapeutic techniques and therapist skillfulness)과 유의하게 연관되는가에 대한 연구이다. 단기 역동 정신치료가 정신치 료적 기술과 치료자의 능숙함과 연관된다는 보고들이 있다 (Crits-Christoph와 Connolly 1999; Messer 2001). 정신치료적 기술에는 해석의 정확성(accuracy of interpretation)(CritsChristoph 등 1988), 치료 계획에 대한 치료자의 개입의 일관 성(adherence of therapists' interventions to the 'plan')(Messer 등 1992), 표현적 기법의 능숙한 전달(competent delivery of expressive, but not of supportive techniques)(Barber 등 1996) 등이 보고되었다. 하지만 상대적으로 정신역동적 기 법들의 횟수(frequency of psychodynamic techniques)가 치 료 결과와 연관이 된다는 증거들은 거의 없다고 보고되기도 하였다(Crits-Christoph와 Connolly 1999).

둘째, 전이 양상(transference patterns)의 특정한 변화들 이 정신역동 정신치료의 결과와 유의하게 연관되는가에 대 한 연구이다. 일반적으로는 전이의 해석이 정신분석이나 정 신역동적 정신치료의 핵심적인 요소라고 여겨지고는 있으나 (Strachey 1969; Gabbard와 Westen 2003), 많은 연구에서 전 이 해석과 치료 결과 사이에는 다소 부정적인 연관성이 있는 것으로 보고하였다(Høglend와 Piper 1995; Connolly 등 1999; Ogrodniczuk와 Piper 1999). 단기 역동 정신치료에서 전이 해석의 횟수는 부정적인 치료 결과나 치료 관계와 연관이 된다고 보고되었는데, 특히 대상 관계의 질이 낮게 평가되는 환자들에서 그러하였다. 대상 관계의 질이 높게 평가된 환자 에게도 낮거나 중등도의 전이 해석은 다소 효과적이었으나 
높은 수준의 전이 해석은 도움이 되지 않는 것 같다고 보고 하였다(Piper 등 1991a; Piper 등 1991b; Connolly 등 1999). 하나의 연구에서는 반대적인 결과를 보고하였는데, Høglend 등(2006)은 비슷한 환자군에서 장기적 역동 정신치료를 세 분하여 전이(transference) 해석을 하는 군과 하지 않는 군으 로 나누어 무작위 배정한 뒤 결과를 확인하였다. 초기 분석 에서 큰 차이를 발견하지는 못하였으나, 성숙한 대상 관계를 가지지 못한 환자군에서 전이 해석이 도움이 되는 양상을 보 였다고 하였다. 그리고 이 효과는 장기적으로 유지되었다고 보고하였다(Høglend 등 2008). 이렇게 전이 해석에 대한 연 구 결과가 일관적이지 못한 이유를 추정해 보면 다음과 같 다. 전이 해석은 적절한 시기에 효과적으로 사용하면 치료적 도움이 되지만, 잘 다루지 못하면 부정적 전이 양상이 발전 될 수 있다. Gabbard(2006)는 전이 해석의 타이밍이 매우 중 요하다고 강조하며 다른 중재 기법들보다도 치료적 동맹에 큰 영향을 가진다고 하였다. 공감, 지지, 그리고 타당성(validation) 등이 환자가 전이 해석을 받아들이는 데 필수적이다. 외과 의사에게 수술 전에 마취가 꼭 필요하듯이, 정신분석가 는 해석 전에 환경, 즉 치료적 동맹을 조성하는 것이 필요한 것이다. 또한 그는 대상 관계의 질이 떨어지는 환자들은 치 료자를 믿을 수 있는 조력자로 보기가 어려운데, 치료적 동 맹에 대해 직접적으로 설명해주고, 환자의 전이에서 보이는 왜곡을 해석하는 과정이 있으면, 환자의 치료적 동맹은 강화 될 수 있고 건설적인 방향으로 치료적 작업이 지속될 수 있 다고 하였다(Gabbard 2006).

셋째, 치료적 동맹(helping alliance)이 정신역동적 정신치 료의 치료 결과의 유의한 예측 인자인가에 대해서이다. 이는 여러 연구들에서 밝혀져 왔으며(Stiles 등 1998; Crits-Christoph와 Connolly 1999; Barber 등 2000; Messer 2001), 한 연 구에서는 치료적 동맹이 정확한 해석과도 유의한 관계를 가 진다고 보고하였다(Crits-Christoph와 Connolly 1999). 한 메 타 분석에서는 치료적 동맹의 강도가 치료 결과에 가장 중 요한 요소임을 주장하였다(Martin 등 2000).

마지막으로, 용량-반응 (dose-effect) 관계에 대한 이슈이 다. 즉, 정신치료의 세션 횟수와 치료 효과와의 관계에 대한 연구들이 있다. 우선, 치료 길이에 대한 연구 결과들은, 환자 에게 인격적인 문제(personality difficulties)가 있는 경우라 면 치료 기간이 길수록 좋다는 결과들이 보고되었다(Doidge 1997). 한 주에 시행되는 치료의 횟수에 대한 연구로는, 세 개 의 연구가 한 주에 4 5회 진행되는 정신분석과 한 주에 1 2 회 진행되는 정신분석적 정신치료를 비교했을 때, 세 연구 모 두에서 정신분석이 나은 것으로 보고되었다(Doidge 1997). The Columbia Group에서는 자발적으로 정신분석에서 정
신분석적 치료로 전환한 군이 전환하지 않고 정신분석을 유 지했던 군에 비해 효과가 부족했다(Weber 등 1985a). 이는 신경증 환자뿐 아니라 심한 인격 문제를 가진 $\mathrm{BPD}$ 환자를 대 상으로 한 연구에서도 치료 종료 시점에서 정신분석이 보다 나은 결과를 보여 주었다고 보고하였다(Waldinger와 Gunderson 1984).

이러한 정신치료 효과와 관련하여 생각할 수 있는 이슈들 은 꾸준히 연구가 되고 있으며, 결과들이 속속 보고되고 있다.

\section{결 론}

정신치료는 그 특성상 효과를 검증하기 어려운 경우가 많 다. 특히, 정신분석이나 장기 역동적 정신치료의 경우 제한 된 환경에서 장기간 연구가 진행되는 것은 어렵다. 그러나, 정신역동적 정신치료에 대한 $\mathrm{RCT}$ 가 없는 것은 아니며, 특 히 단기 역동적 정신치료는 여러 질환에 걸쳐 연구가 보고 되었다. 이러한 결과들을 종합해 볼 때 정신분석적 정신치료 가 적어도 다른 약물치료나 CBT에 비해 치료 효과 연구가 적은 것은 아닌 것으로 추정해 볼 수 있다. 또한, 정신분석과 정신분석적 정신치료의 경우 질환의 증상 호전 측면 외에 삶의 질이나 적응 능력, 그리고 정신 내적 측면의 변화 등에 효과가 있는 것으로 생각된다. 정신분석 이론 및 방법을 적용 한 단기 역동적 정신치료를 비롯한 정신분석적 정신치료가 대체로 효과가 있는 것으로 유추해 볼 때, 더 장기적이고 집 중적인(intensive) 정신분석도, 비록 RCT 연구가 어려워서 직접 확인은 어렵지만 마찬가지로 효과가 있을 것으로 유추 해 볼 수 있다. 향후에도 정신분석과 정신분석적 정신치료에 대한 좀 더 근거 있는 효과 연구가 필요할 것으로 생각하며, 아울러 뇌영상 연구 등을 통해 정신치료 기전 및 현상에 대 한 과학적 입증이 필요할 것으로 생각한다.

\section{Conflicts of Interest}

The authors have no financial conflicts of interest.

\section{REFERENCES}

Abbass A, Driessen E. The efficacy of short-term psychodynamic psychotherapy for depression: a summary of recent findings. Acta Psychiatr Scand 2010;121:398; author reply 398-399.

Abbass A, Kisely S, Kroenke K. Short-term psychodynamic psychotherapy for somatic disorders. Systematic review and meta-analysis of clinical trials. Psychother Psychosom 2009;78:265-274.

Abbass A, Sheldon A, Gyra J, Kalpin A. Intensive short-term dynamic psychotherapy for DSM-IV personality disorders: a randomized controlled trial. J Nerv Ment Dis 2008;196:211-216.

Ablon JS, Jones EE. On analytic process. J Am Psychoanal Assoc 2005; 53:541-568; discussion 569-578.

Bachar E, Latzer Y, Kreitler S, Berry EM. Empirical comparison of two psychological therapies. Self psychology and cognitive orientation in 
the treatment of anorexia and bulimia. J Psychother Pract Res 1999; 8:115-128.

Bachrach HM, Galatzer-Levy R, Skolnikoff A, Waldron S Jr. On the efficacy of psychoanalysis. J Am Psychoanal Assoc 1991;39:871-916.

Barber JP, Barrett MS, Gallop R, Rynn MA, Rickels K. Short-term dynamic psychotherapy versus pharmacotherapy for major depressive disorder: a randomized, placebo-controlled trial. J Clin Psychiatry 2012;73:66-73.

Barber JP, Connolly MB, Crits-Christoph P, Gladis L, Siqueland L. Alliance predicts patients' outcome beyond in-treatment change in symptoms. J Consult Clin Psychol 2000;68:1027-1032.

Barber JP, Crits-Christoph P, Luborsky L. Effects of therapist adherence and competence on patient outcome in brief dynamic therapy. J Consult Clin Psychol 1996;64:619-622.

Barkham M, Rees A, Shapiro DA, Stiles WB, Agnew RM, Halstead J, et al. Outcomes of time-limited psychotherapy in applied settings: replicating the Second Sheffield Psychotherapy Project. J Consult Clin Psychol 1996;64:1079-1085.

Bateman A, Fonagy P. Effectiveness of partial hospitalization in the treatment of borderline personality disorder: a randomized controlled trial. Am J Psychiatry 1999;156:1563-1569.

Bateman A, Fonagy P. Randomized controlled trial of outpatient mentalization-based treatment versus structured clinical management for borderline personality disorder. Am J Psychiatry 2009;166:1355-1364.

Bateman A, Fonagy P. Treatment of borderline personality disorder with psychoanalytically oriented partial hospitalization: an 18-month follow-up. Am J Psychiatry 2001;158:36-42.

Benson K, Hartz AJ. A comparison of observational studies and randomized, controlled trials. N Engl J Med 2000;342:1878-1886.

Beutel ME, Rasting M, Stuhr U, Rüger B, Leuzinger-Bohleber M. Assessing the impact of psychoanalyses and long-term psychoanalytic therapies on health care utilization and costs. Psychother Res 2004; 14:146-160.

Beutler LE. Identifying empirically supported treatments: what if we didn't? J Consult Clin Psychol 1998;66:113-120.

Blatt SJ. The differential effect of psychotherapy and psychoanalysis with anaclitic and introjective patients: the Menninger Psychotherapy Research Project revisited. J Am Psychoanal Assoc 1992;40:691-724.

Blatt SJ, Auerbach JS. Psychodynamic measures of therapeutic change. Psychoanal Inq 2003;23:268-307.

Blatt SJ, Shahar G. Psychoanalysis--with whom, for what, and how? Comparisons with psychotherapy. J Am Psychoanal Assoc 2004;52: 393-447.

Blomberg J, Lazar A, Sandell R. Long-term outcome of long-term psychoanalytically oriented therapies: first findings of the stockholm outcome of psychotherapy and psychoanalysis study. Psychother Res 2001;11:361-382.

Bögels SM, Wijts P, Oort FJ, Sallaerts SJ. Psychodynamic psychotherapy versus cognitive behavior therapy for social anxiety disorder: an efficacy and partial effectiveness trial. Depress Anxiety 2014;31:363-373.

Bressi C, Porcellana M, Marinaccio PM, Nocito EP, Magri L. Shortterm psychodynamic psychotherapy versus treatment as usual for depressive and anxiety disorders: a randomized clinical trial of efficacy. J Nerv Ment Dis 2010;198:647-652.

Brockmann J, Schlüter T, Eckert J. [Therapy goals, change of goals and goal attainment in the process of psychoanalytically oriented and behavior long-term therapy--a comparative study from the private practices of insurance-registered psychotherapists]. Psychother Psychosom Med Psychol 2003;53:163-170.

Brom D, Kleber RJ, Defares PB. Brief psychotherapy for posttraumatic stress disorders. J Consult Clin Psychol 1989;57:607-612.

Busch FN, Milrod BL, Sandberg LS. A study demonstrating efficacy of a psychoanalytic psychotherapy for panic disorder: implications for psychoanalytic research, theory, and practice. J Am Psychoanal Assoc 2009;57:131-148.
Chambless DL, Baker MJ, Baucom DH, Beutler LE, Calhoun KS, Crits-Christoph P, et al. Update on empirically validated therapies, II. Clin Psychol 1998;51:3-16.

Chambless DL, Hollon SD. Defining empirically supported therapies. J Consult Clin Psychol 1998;66:7-18.

Chambless DL, Ollendick TH. Empirically supported psychological interventions: controversies and evidence. Annu Rev Psychol 2001; 52:685-716.

Clarkin JF, Levy KN, Lenzenweger MF, Kernberg OF. Evaluating three treatments for borderline personality disorder: a multiwave study. Am J Psychiatry 2007;164:922-928.

Cohen J. Statistical power analysis for the behavioral sciences. Hillsdale, NJ: Lawrence Erlbaum Associates; 1988.

Concato J, Shah N, Horwitz RI. Randomized, controlled trials, observational studies, and the hierarchy of research designs. N Engl J Med 2000;342:1887-1892.

Connolly M, Crits-Christoph P, Shappell S, Barber J, Luborsky L, Shaffer C. Relation of transference interpretations to outcome in the early sessions of brief supportive-expressive psychotherapy. Psychother Res 1999;9:485-495.

Creed F, Fernandes L, Guthrie E, Palmer S, Ratcliffe J, Read N, et al. The cost-effectiveness of psychotherapy and paroxetine for severe irritable bowel syndrome. Gastroenterology 2003;124:303-317.

Crits-Christoph P, Connolly MB. Alliance and technique in short-term dynamic therapy. Clin Psychol Rev 1999;19:687-704.

Crits-Christoph P, Cooper A, Luborsky L. The accuracy of therapists' interpretations and the outcome of dynamic psychotherapy. J Consult Clin Psychol 1988;56:490-495.

Crits-Christoph P, Gibbons MBC, Narducci J, Schamberger M, Gallop R. Interpersonal problems and the outcome of interpersonally oriented psychodynamic treatment of GAD. Psychotherapy 2005;42:211-224.

Crits-Christoph P, Siqueland L, McCalmont E, Weiss RD, Gastfriend DR, Frank A, et al. Impact of psychosocial treatments on associated problems of cocaine-dependent patients. J Consult Clin Psychol 2001; 69:825-830.

Cuijpers P, van Straten A, Andersson G, van Oppen P. Psychotherapy for depression in adults: a meta-analysis of comparative outcome studies. J Consult Clin Psychol 2008;76:909-922.

Dare C, Eisler I, Russell G, Treasure J, Dodge L. Psychological therapies for adults with anorexia nervosa: randomised controlled trial of out-patient treatments. Br J Psychiatry 2001;178:216-221.

Doering S, Hörz S, Rentrop M, Fischer-Kern M, Schuster P, Benecke C, et al. Transference-focused psychotherapy v. treatment by community psychotherapists for borderline personality disorder: randomised controlled trial. Br J Psychiatry 2010;196:389-395.

Doidge N. Empirical evidence for the efficacy of psychoanalytic psychotherapies and psychoanalysis: an overview. Psychoanal Inq 1997;17: 102-150.

Driessen E, Cuijpers P, de Maat SC, Abbass AA, de Jonghe F, Dekker JJ. The efficacy of short-term psychodynamic psychotherapy for depression: a meta-analysis. Clin Psychol Rev 2010;30:25-36.

Dührssen A, Jorswieck E. [An empirical-statistical study of the effectiveness of psychoanalytic treatment]. Z Psychosom Med Psychoanal 1965;36:166-169

Ehlers A, Bisson J, Clark DM, Creamer M, Pilling S, Richards D, et al. Do all psychological treatments really work the same in posttraumatic stress disorder? Clin Psychol Rev 2010;30:269-276.

Ekeblad A, Falkenström F, Andersson G, Vestberg R, Holmqvist R. Randomized trial of interpersonal psychotherapy and cognitive behavioral therapy for major depressive disorder in a community-based psychiatric outpatient clinic. Depress Anxiety 2016 Mar 31 [Epub]. http://dx.doi.org/10.1002/da.22495.

Emmelkamp PM, Benner A, Kuipers A, Feiertag GA, Koster HC, van Apeldoorn FJ. Comparison of brief dynamic and cognitive-behavioural therapies in avoidant personality disorder. Br J Psychiatry 2006; 
189:60-64

Erle JB. An approach to the study of analyzability and analyses: the course of forty consecutive cases selected for supervised analysis. Psychoanal Q 1979;48:198-228.

Erle JB, Goldberg DA. Observations on assessment of analyzability by experienced analysts. J Am Psychoanal Assoc 1984;32:715-737.

Fairburn CG. Evidence-based treatment of anorexia nervosa. Int J Eat Disord 2005;37 Suppl:S26-S30; discussion S41-S42.

Fairburn CG, Kirk J, O'Connor M, Cooper PJ. A comparison of two psychological treatments for bulimia nervosa. Behav Res Ther 1986;24: 629-643.

Fairburn CG, Norman PA, Welch SL, O'Connor ME, Doll HA, Peveler RC. A prospective study of outcome in bulimia nervosa and the longterm effects of three psychological treatments. Arch Gen Psychiatry 1995;52:304-312.

Gabbard GO. Long-term psychodynamic psychotherapy: a basic text. Arlington, VA: American Psychiatric Pub;2010.

Gabbard GO. When is transference work useful in dynamic psychotherapy? Am J Psychiatry 2006;163:1667-1669.

Gabbard GO, Westen D. Rethinking therapeutic action. Int J Psychoanal 2003;84(Pt 4):823-841.

Gallagher-Thompson D, Steffen AM. Comparative effects of cognitivebehavioral and brief psychodynamic psychotherapies for depressed family caregivers. J Consult Clin Psychol 1994;62:543-549.

Garner DM, Rockert W, Davis R, Garner MV, Olmsted MP, Eagle M. Comparison of cognitive-behavioral and supportive-expressive therapy for bulimia nervosa. Am J Psychiatry 1993;150:37-46.

Giesen-Bloo J, van Dyck R, Spinhoven P, van Tilburg W, Dirksen C, van Asselt T, et al. Outpatient psychotherapy for borderline personality disorder: randomized trial of schema-focused therapy vs transference-focused psychotherapy. Arch Gen Psychiatry 2006;63:649-658.

Gowers S, Norton K, Halek C, Crisp AH. Outcome of outpatient psychotherapy in a random allocation treatment study of anorexia nervosa. Int J Eat Disord 1994;15:165-177.

Gregory RJ, Chlebowski S, Kang D, Remen AL, Soderberg MG, Stepkovitch J, et al. A controlled trial of psychodynamic psychotherapy for co-occurring borderline personality disorder and alcohol use disorder. Psychotherapy (Chic) 2008;45:28-41.

Gunderson JG, Gabbard GO. Making the case for psychoanalytic therapies in the current psychiatric environment. J Am Psychoanal Assoc 1999;47:679-704; discussion 704-740.

Guthrie E, Creed F, Dawson D, Tomenson B. A controlled trial of psychological treatment for the irritable bowel syndrome. Gastroenterology 1991;100:450-457.

Hamilton J, Guthrie E, Creed F, Thompson D, Tomenson B, Bennett R, et al. A randomized controlled trial of psychotherapy in patients with chronic functional dyspepsia. Gastroenterology 2000;119:661-669.

Hellerstein DJ, Rosenthal RN, Pinsker H, Samstag LW, Muran JC, Winston A. A randomized prospective study comparing supportive and dynamic therapies. Outcome and alliance. J Psychother Pract Res 1998;7:261-271.

Høglend P, Amlo S, Marble A, Bøgwald KP, Sørbye O, Sjaastad MC, et al. Analysis of the patient-therapist relationship in dynamic psychotherapy: an experimental study of transference interpretations. Am J Psychiatry 2006;163:1739-1746.

Høglend P, Bøgwald KP, Amlo S, Marble A, Ulberg R, Sjaastad MC, et al. Transference interpretations in dynamic psychotherapy: do they really yield sustained effects? Am J Psychiatry 2008;165:763-771.

Høglend P, Piper WE. Focal adherence in brief dynamic psychotherapy: a comparison of findings from two independent studies. Psychotherapy 1995;32:618-628.

Johansson R, Ekbladh S, Hebert A, Lindström M, Möller S, Petitt E, et al. Psychodynamic guided self-help for adult depression through the internet: a randomised controlled trial. PLoS One 2012;7:e38021.

Jones EE. Therapeutic action: a guide to psychoanalytic therapy. North- vale, NJ: Rowman \& Littlefield;2000.

Jørgensen CR, Freund C, Bøye R, Jordet H, Andersen D, Kjølbye M. Outcome of mentalization-based and supportive psychotherapy in patients with borderline personality disorder: a randomized trial. Acta Psychiatr Scand 2013;127:305-317.

Kernberg OF, Burstein ED, Coyne L, Appelbaum A, Horwitz L, Voth H. Psychotherapy and psychoanalysis: final report of the Menninger Foundation's psychotherapy research project. Bull Menninger Clin 1972;36:1-277.

Kernberg OF, Yeomans FE, Clarkin JF, Levy KN. Transference focused psychotherapy: overview and update. Int J Psychoanal 2008;89:601620.

Knekt P, Lindfors O, Härkänen T, Välikoski M, Virtala E, Laaksonen MA, et al. Randomized trial on the effectiveness of long-and shortterm psychodynamic psychotherapy and solution-focused therapy on psychiatric symptoms during a 3-year follow-up. Psychol Med 2008; 38:689-703.

Knijnik DZ, Kapczinski F, Chachamovich E, Margis R, Eizirik CL. [Psychodynamic group treatment for generalized social phobia]. Rev Bras Psiquiatr 2004;26:77-81.

Lamb WK. A Meta-analysis of outcome studies in long-term psychodynamic psychotherapy and psychoanalysis. Oakland, CA: University of California, Berkeley;2004.

Leichsenring F. Are psychodynamic and psychoanalytic therapies effective?: a review of empirical data. Int J Psychoanal 2005;86(Pt 3):841868.

Leichsenring F. Randomized controlled versus naturalistic studies: a new research agenda. Bull Menninger Clin 2004;68:137-151.

Leichsenring F, Biskup J, Kreische R, Staats H. The Göttingen study of psychoanalytic therapy: first results. Int J Psychoanal 2005;86(Pt 2): 433-455.

Leichsenring F, Klein S, Salzer S. The efficacy of psychodynamic psychotherapy in specific mental disorders: a 2013 update of empirical evidence. Contemp Psychoanal 2014;50:89-130.

Leichsenring F, Leibing E. Cognitive-behavioural therapy for avoidant personality disorder. Br J Psychiatry 2007;190:80; author reply 80-81.

Leichsenring F, Leibing E. The effectiveness of psychodynamic therapy and cognitive behavior therapy in the treatment of personality disorders: a meta-analysis. Am J Psychiatry 2003;160:1223-1232.

Leichsenring F, Salzer S, Beutel ME, Herpertz S, Hiller W, Hoyer J, et al. Psychodynamic therapy and cognitive-behavioral therapy in social anxiety disorder: a multicenter randomized controlled trial. Am J Psychiatry 2013;170:759-767.

Leichsenring F, Salzer S, Jaeger U, Kächele H, Kreische R, Leweke F, et al. Short-term psychodynamic psychotherapy and cognitive-behavioral therapy in generalized anxiety disorder: a randomized, controlled trial. Am J Psychiatry 2009;166:875-881.

Leuzinger-Bohleber M, Stuhr U, Rüger B, Beutel M. How to study the 'quality of psychoanalytic treatments' and their long-term effects on patients' well-being: a representative, multi-perspective follow-up study. Int J Psychoanal 2003;84(Pt 2):263-290.

Lipton SD. The last hour. J Am Psychoanal Assoc 1961;9:325-330.

Luborsky L, Crits-Christoph P, Mintz J, Auerbach A. Who will benefit from psychotherapy?: predicting therapeutic outcomes. New York: Basic Books; 1988. p.1-416.

Luborsky L, Diguer L, Seligman DA, Rosenthal R, Krause ED, Johnson S, et al. The researcher's own therapy allegiances: a "wild card" in comparisons of treatment efficacy. Clin Psychol Sci Pract 1999;6:95106.

Maina G, Forner F, Bogetto F. Randomized controlled trial comparing brief dynamic and supportive therapy with waiting list condition in minor depressive disorders. Psychother Psychosom 2005;74:43-50.

Martin DJ, Garske JP, Davis MK. Relation of the therapeutic alliance with outcome and other variables: a meta-analytic review. J Consult Clin Psychol 2000;68:438-450. 
Messer SB. What makes brief psychodynamic therapy time efficient. Clin Psychol Sci Pract 2001;8:5-22.

Messer SB, Tishby O, Spillman A. Taking context seriously in psychotherapy research: relating therapist interventions to patient progress in brief psychodynamic therapy. J Consult Clin Psychol 1992;60:678688.

Milrod B, Leon AC, Busch F, Rudden M, Schwalberg M, Clarkin J, et al. A randomized controlled clinical trial of psychoanalytic psychotherapy for panic disorder. Am J Psychiatry 2007;164:265-272.

Monsen K, Monsen JT. Chronic pain and psychodynamic body therapy: a controlled outcome study. Psychotherapy 2000;37:257-269.

Munroe-Blum H, Marziali E. A controlled trial of short-term group treatment for borderline personality disorder. J Pers Disord 1995;9:190-198.

Muran JC, Safran JD, Samstag LW, Winston A. Evaluating an alliancefocused treatment for personality disorders. Psychotherapy 2005;42: 532-545.

Nathan PE, Gorman JM. A guide to treatments that work. New York: Oxford University Press;2015. p.1-54.

Ogrodniczuk JS, Piper WE. Use of transference interpretations in dynamically oriented individual psychotherapy for patients with personality disorders. J Pers Disord 1999;13:297-311.

Piper WE, Azim HF, Joyce AS, McCallum M. Transference interpretations, therapeutic alliance, and outcome in short-term individual psychotherapy. Arch Gen Psychiatry 1991a;48:946-953.

Piper WE, Azim HF, Joyce AS, McCallum M, Nixon GW, Segal PS. Quality of object relations versus interpersonal functioning as predictors of therapeutic alliance and psychotherapy outcome. J Nerv Ment Dis 1991b;179:432-438.

Rossouw TI, Fonagy P. Mentalization-based treatment for self-harm in adolescents: a randomized controlled trial. J Am Acad Child Adolesc Psychiatry 2012;51:1304-1313.e3.

Rudolf G, Dilg R, Grande T, Jacobsen T, Keller W. Effectiveness and efficiency of long-term psychoanalytic therapy: the practice study of long-term psychoanalytic therapy. In: Gerlach A, Springer AG, Schlösser A. Psychoanalyse des glaubens. Giessen: Psychosozial Verlag; 2004. p.515-528

Rudolf G, Manz R, Ori C. [Results of psychoanalytic therapy]. Z Psychosom Med Psychoanal 1994;40:25-40.

Salminen JK, Karlsson H, Hietala J, Kajander J, Aalto S, Markkula J, et al. Short-term psychodynamic psychotherapy and fluoxetine in major depressive disorder: a randomized comparative study. Psychother Psychosom 2008;77:351-357.

Sandahl C, Herlitz K, Ahlin G, Rönnberg S. Time-limited group psychotherapy for moderately alcohol dependent patients: a randomized controlled clinical trial. Psychother Res 1998;8:361-378.

Sandell R. Wiederholte langzeitkatamnesen von langzeit- psychotherapien und psychoanalysen. Z Psychosom Med Psychother 1999;45:43-56.

Sandell R, Blomberg J, Lazar A, Carlsson J, Broberg J, Schubert J. Varieties of long-term outcome among patients in psychoanalysis and long-term psychotherapy: a review of findings in the Stockholm Outcome of Psychoanalysis and Psychotherapy Project (STOPP). Int J Psychoanal 2000;81(Pt 5):921-942.

Sattel H, Lahmann C, Gündel H, Guthrie E, Kruse J, Noll-Hussong $\mathbf{M}$, et al. Brief psychodynamic interpersonal psychotherapy for patients with multisomatoform disorder: randomised controlled trial. Br J Psychiatry 2012;200:60-67.

Seligman ME. The effectiveness of psychotherapy. The Consumer Reports study. Am Psychol 1995;50:965-974.

Shadish WR, Cook TD, Campbell DT. Experimental and quasi-experimental designs for generalized causal inference. Boston: Houghton Mifflin;2002
Shapiro DA, Barkham M, Rees A, Hardy GE, Reynolds S, Startup M. Effects of treatment duration and severity of depression on the effectiveness of cognitive-behavioral and psychodynamic-interpersonal psychotherapy. J Consult Clin Psychol 1994;62:522-534.

Stiles WB, Agnew-Davies R, Hardy GE, Barkham M, Shapiro DA. Relations of the alliance with psychotherapy outcome: findings in the Second Sheffield Psychotherapy Project. J Consult Clin Psychol 1998;66:791-802.

Strachey J. The nature of the therapeutic action of psychoanalysis. Int J Psychoanal 1969;50:275-292.

Svartberg M, Stiles TC, Seltzer MH. Randomized, controlled trial of the effectiveness of short-term dynamic psychotherapy and cognitive therapy for cluster C personality disorders. Am J Psychiatry 2004; $161: 810-817$

Tasca GA, Ritchie K, Conrad G, Balfour L, Gayton J, Lybanon V, et al. Attachment scales predict outcome in a randomized controlled trial of two group therapies for binge eating disorder: an aptitude by treatment interaction. Psychother Res 2006;16:106-121.

Thompson LW, Gallagher D, Breckenridge JS. Comparative effectiveness of psychotherapies for depressed elders. J Consult Clin Psychol 1987;55:385-390.

Town JM, Abbass A, Hardy G. Short-term psychodynamic psychotherapy for personality disorders: a critical review of randomized controlled trials. J Pers Disord 2011;25:723-740.

Vinnars B, Barber JP, Norén K, Gallop R, Weinryb RM. Manualized supportive-expressive psychotherapy versus nonmanualized community-delivered psychodynamic therapy for patients with personality disorders: bridging efficacy and effectiveness. Am J Psychiatry 2005; 162:1933-1940.

Waldinger RJ, Gunderson JG. Completed psychotherapies with borderline patients. Am J Psychother 1984;38:190-202.

Wallerstein RS. Forty-two lives in treatment: a study of psychoanalysis and psychotherapy. New York: The Analytic Press;2000. p.1-768.

Weber JJ, Bachrach HM, Solomon M. Factors associated with the outcome of psychoanalysis: report of the Columbia Psychoanalytic Center Research Project: II. Int Rev Psychoanal 1985a;12:127-141.

Weber JJ, Bachrach HM, Solomon M. Factors associated with the outcome of psychoanalysis: report of the Columbia Psychoanalytic Center Research Project: III. Int Rev Psychoanal 1985b;12:251-262.

Weber JJ, Solomon M, Bachrach HM. Characteristics of psychoanalytic clinic patients: report of the Columbia Psychoanalytic Center Research Project: I. Int Rev Psychoanal 1985c;12:13-26.

Westen D, Novotny CM, Thompson-Brenner H. The empirical status of empirically supported psychotherapies: assumptions, findings, and reporting in controlled clinical trials. Psychol Bull 2004;130:631-663.

Winston A, Laikin M, Pollack J, Samstag LW, McCullough L, Muran JC. Short-term psychotherapy of personality disorders. Am J Psychiatry 1994;151:190-194.

Woody G, Luborsky L, McLellan AT, O'Brien CP. Corrections and revised analyses for psychotherapy in methadone maintenance patients. Arch Gen Psychiatry 1990;47:788-789.

Woody GE, Luborsky L, McLellan AT, O'Brien CP, Beck AT, Blaine J, et al. Psychotherapy for opiate addicts. Does it help? Arch Gen Psychiatry 1983;40:639-645.

Woody GE, McLellan AT, Luborsky L, O'Brien CP. Psychotherapy in community methadone programs: a validation study. Am J Psychiatry 1995; 152:1302-1308

Zobel I, Kech S, van Calker D, Dykierek P, Berger M, Schneibel R, et al. Long-term effect of combined interpersonal psychotherapy and pharmacotherapy in a randomized trial of depressed patients. Acta Psychiatr Scand 2011;123:276-282. 